\title{
Evaluar la competencia "Innovación, Creatividad y Emprendimiento" en asignaturas del área de la Mecánica de los Fluidos. Caso de estudio en Grado y Máster
}

\section{P. Amparo López-Jiménez ${ }^{\mathrm{a}}$, Modesto Pérez-Sánchez ${ }^{\mathrm{a}}$}

${ }^{a}$ Departamento de Ingeniería Hidráulica y Medio Ambiente. Universitat Politècnica de València. palopez@upv.es; mopesan1@upv.es.

\begin{abstract}
The UPV generic student outcomes "Innovation, creativity and entrepreneurship" belongs to the strategic plan of the UPV to reach all transversal competences, being in itself a competence to be used as a learning strategy for students to fix complex contents in a deductive way. This contribution describes the experience of the authors in the implementation of this competence in different Bachelor and Master's Degrees, in matters related to Fluid Mechanics. Different activities 6-3-5 were performed in these levels with groups of very diverse students, with a similar programming of the activity. The experience allowed the authors affirm that this strategy is a powerful technique for students to develop their creativity, as well as their curiosity for acquiring the knowledge. The reach of the knowledge is finally expected of the curricular content of the subjects at both levels. Two rubbrics were developed, which enable to evaluate the domain level with various indicators considering weighted values each activity.
\end{abstract}

Keywords: UPV generic student outcomes, creativity, innovation, entrepreneurship, fluid mechanics, dispersion of pollutants

\footnotetext{
Resumen

La competencia transversal "Innovación, creatividad y emprendimiento" pertenece al plan estratégico de la UPV para alcanzar todas las competencias transversales, siendo en sí misma una competencia que puede ser utilizada tambien como estrategia de aprendizaje para que los alumnos fijen contenidos complejos de una forma deductiva. La presente contribución describe la experiencia de los autores en la implementación de esta competencia a nivel de Grado y Master en diferentes titulaciones, en materias relacionadas con la Mecánica de Fluidos. La realización de actividades 6-3-5 en estos niveles, con grupos de alumnos diversos, con una programación similar de la actividad, permite afirmar que es una técnica potente para que los alumnos desarrollen su creatividad a la vez que su curiosidad para adquirir los conocimientos que finalmente se esperan del contenido curricular de las asignaturas en ambos niveles. Se presenta una rúbrica para ambos dominios que permite evaluar varios indicadores con diferentes pesos ponderados cada actividad propuesta.
} 
Palabras clave: competencia transversal, creatividad, innovación, emprendimiento, mecánica de fluidos, dispersión de contaminantes

\section{Introducción}

La creatividad, innovación y emprendimiento son conceptos que están fuertemente ligados a la ingeniería, en la cual, la búsqueda de soluciones a un problema así como adoptar la solución definitiva son actividades y funciones que un ingeniero debe adoptar en su día a día. No obstante, aunque los tres términos pueden conducir a pensar que son sinónimos, existen diferencias entre ellos. Por ello, si atendemos a su definición (RAE, 2017) de los verbos:

- Crear: "Establecrr, fundar intr-ducir por vez -rimer algo"

- Innovar: "Mudar o alterar algo, introduciendo novedades"

- Emprender: "Acometer y comenzar una obra"

Atendiendo a las definiciones anteriormente expuestas, se pone de manifiesto que aunque se encuentran relacionados no son sinónimos, existiendo una correlación temporal en la toma de decisiones entre las acciones de creatividad, innovación y emprendimiento. Aunque en un principio, estos conceptos pueden ser asociados a temáticas empresariales, el trinomio definido anteriormente esta asociado a cualquier ámbito de la vida social y profesional. Por tanto, la sociedad necesita de gente con un espíritu emprendedor (Fernandes et al., 2017), la cual innove soluciones a los problemas que presente la sociedad, emprendiendo ideas que conduzcan a crear nuevos productos, servicios y/o propuestas que mejoren a las existentes.

La Universidad debe ser pionera en la mejora de estos profesionales y por tanto, ser parte de la unidad formadora en los estudiantes que cursan los estudios de grado y/o máster. Estos alumnos se encuentran en una fase terminal de formación, y en un breve periodo corto de tiempo, se encontraran con situaciones en las cuales, deberán detectar el problema, buscar posibles soluciones y emprender la que se considere que lo solventa de una forma más éficaz. Relacionado con lo anteriormente expuesto, la Universitat Politècnica de València puso en marcha el proyecto de competencias transversales denominado Plan Estratégico UPV2020, donde el primer reto es "La Universitat Politècnica de València tiene como objetivo avanzar hacia modelos de formación que logren que sus estudiantes adquieran las competencias necesarias para poder tener una adecuada inserción laboral" (Vidal-Carreras et al., 2016). Estas competencias, las cuales suman un total de 13, buscan complementar la formación del estudiante junto con las competencias genéricas y específicas de cada uno de los planes de estudios.

Dentro de esas 13 competencias transversales, se encuentra la competencia tranversal $C T$ 04 “Innovación, Emprendimiento y Creatividad", la cual es trabajada por el alumno a lo largo de su formación universitaria en tres niveles diferentes en los estudios de grado (Nivel I y II) y de máster (Nivel III). Entre las diferentes asignaturas impartidas en el Departamento de Ingeniería Hidráulica y Medio Ambiente, las asignaturas Mecánica de Fluidos (impartida en segundo curso del Grado de Ingeniería Química de la EPSA) y Dispersión de Contaminantes en Agua y Atmósfera (impartida en segundo curso del Máster Universitario en Ingeniería Industrial) son punto de control en Nivel I y III, respectivamente.

Aunque en un principio, puede parecer que esta competencia es difícil de aplicar en asignaturas que no están directamente aplicadas a un mundo empresarial, el presente trabajo muestra que la competencia puede ser desarrollada y evaluada en asignaturas con una fuerte

(cc) EY-NC-ND 2017, Universitat Politècnica de València 
componente teórica o técnica, cómo puede ser las relacionadas con el área de la mecánica de fluidos. Del mismo modo, se muestra la planificación propuesta para desarrollar durante el curso, así como los resultados obtenidos durante una de las evaluaciones desarrolladas.

\section{Objetivos}

El objetivo de la experiencia es desarrollar una estrategia de tareas presenciales y no presenciales, en la cual se muestre cómo se trabaja a lo largo del curso esta competencia, así como, mostrar la metodología propuesta, desarrollada y evaluada en una de las tareas que sirven como envidencia de punto de control.

Finalmente, se analizan las semejanzas y diferencias existentes entre alumnos de nivel de dominio I y de nivel III.

\section{Desarrollo de la innovación}

\subsection{Identificando el problema}

En todos los casos, a lo largo de las diferentes unidades didácticas el docente expone al alumno la metodología de trabajo necesaria para poder resolver un problema. En este caso, la metodología esta centrada en seis intervalos diferentes, los cuales definidos, tal y como se recoge en la Figura 1.

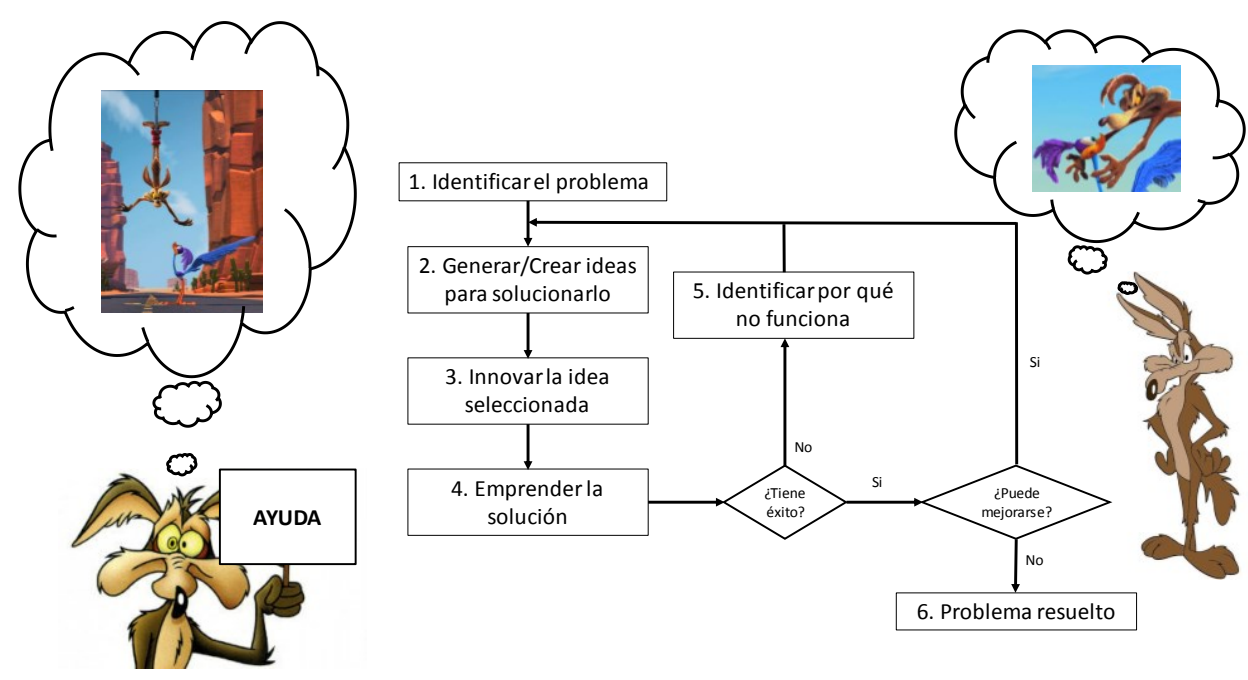

Fig. 1 Identificando el problema y buscando su solución. Elaboración propia

1. Identificando el problema: si se pretende innovar, emprender y crear en cualquier situación que pretenda resolver o mejorar un proceso, el primer paso es detectar el problema. Esta es la primera fase, la cual requiere un análisis previo de la situación. Esta parte de indentificación del problema o deficiencia, está ligada con la competencia transversal CT-03 Análisis y Resolución de Problemas.

2. Generar/Crear ideas: Es la primera de las fases de trabajo, una vez detectado el problema, la búsqueda de ideas que mejoren la situación de partida, constituye uno 
de los procesos claves. Esta búsqueda puede desarrollarse de forma individual o en grupo con diferentes métodos que facilitan el trabajo. Esa generación de ideas será desarrollada en menor o mayor profundidad de acuerdo al nivel de dominio analizado (Figura 2).

3. Innovar la idea seleccionada: Definidas las ideas propuestas, el alumno o grupo de alumnos deben establecer el plan de acción a desarrollar para seleccionar el resultado idóneo, el cual dote de solución al problema planteado. Este plan de acción, en su nivel de dominio I estará centrado unicamente en plasmar las ideas mientras que en un nivel III, los alumnos deben desarrollar el plan de acción a acometer para solventar el problema.

4. Emprender la solución: el trabajo y consecución de los resultados de aprendizaje en los indicadores anteriores (Indicador I y II en la Figura 2), permite al individuo seleccionar la idea (la cual entiende que mejor soluciona o determina el problema planteado), pasando de enumerar el resultado obtenido de la solución (Nivel de dominio I) hasta analizar y/o determinar el valor alcanzado con la solución propuesta en un nivel de dominio III (Figura 2)

5. Identificar el fallo: establecida la solución o idea propuesta, al igual que en cualquier ámbito laboral, debe considerarse si la solución tiene éxito. En caso de que la respuesta sea negativa, el estudiante debe generar o crear nuevas ideas que resuelvan el problema propuesto. En caso de que la respuesta sea positiva, un análisis de la idea adoptada debe ser realizado, con el objeto de buscar nuevas mejoras.

6. Problema resuelto: desarrollado el análisis, sino se considera necesarios adoptar nuevas mejoras, el problema o idea se da por finalizado.

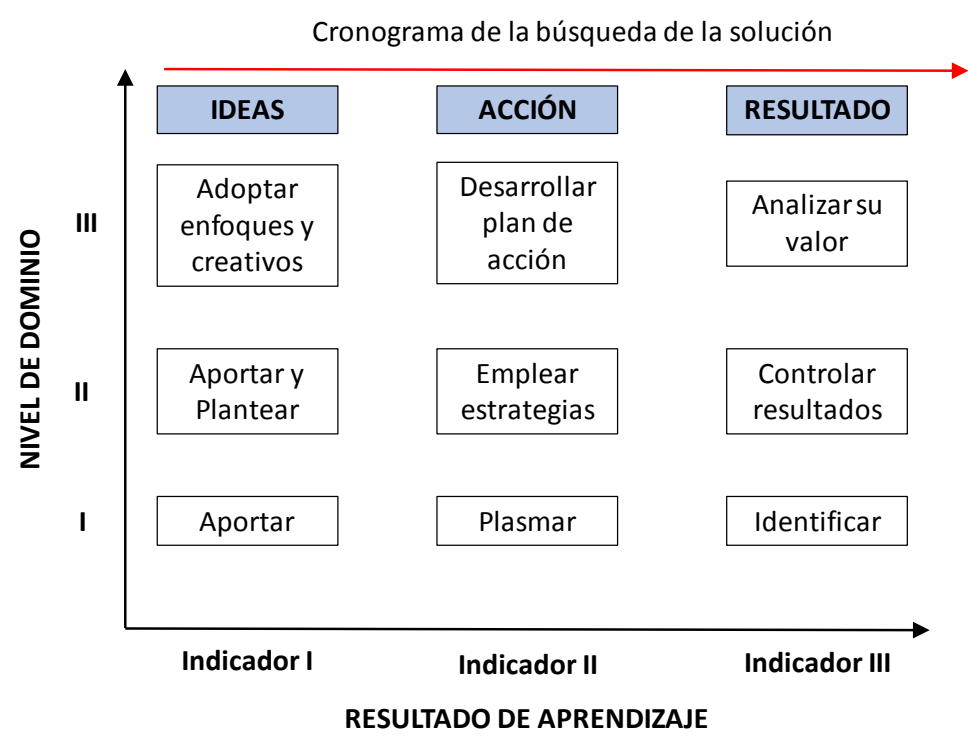

Fig. 2 Resultados de aprendizaje en función del nivel de dominio y cronograma en el tiempo (adaptado de Cuenca et al., 2015)

\subsection{Metodología de trabajo}

(cc) EY-NC-ND 2017, Universitat Politècnica de València 
La competencia transversal ha sido introducida dentro del desarrollo de ambas asignaturas, estableciendo diferentes puntos de control a lo largo de la misma. Estos actos de evaluación permiten medir la evolución de los alumnos a lo largo del curso, a medida que se trabaja.

El trabajo de la competencia ha sido propuesto de una forma lineal y progresiva, en la cual el alumno, guiado por el profesor, trabaja de forma activa la innovación y creatividad en la toma de decisiones. Esta toma de decisiones esta fundamentada en los conceptos desarrollados en cada una de las unidades que se desarrollan en las sesiones presenciales. La creación, innovación y emprendimiento el alumno lo trabaja en las diferentes prácticas de aula e informáticas propuestas y guiadas por el profesor. De forma lateral y no presencial, el alumno puede complementar su formación de forma asíncrona con el uso de objetos de aprendizaje (López-Jimenez \& Pérez-Sánchez, 2016; Pérez-Sánchez \& López-Jimenez, 2017).

Finalmente, se establecen cuatro puntos de control que permiten evaluar la competencia durante el semestre B del curso. Esta evaluación está distribuida temporalmente, tal y como se adjunta en la Figura 3.

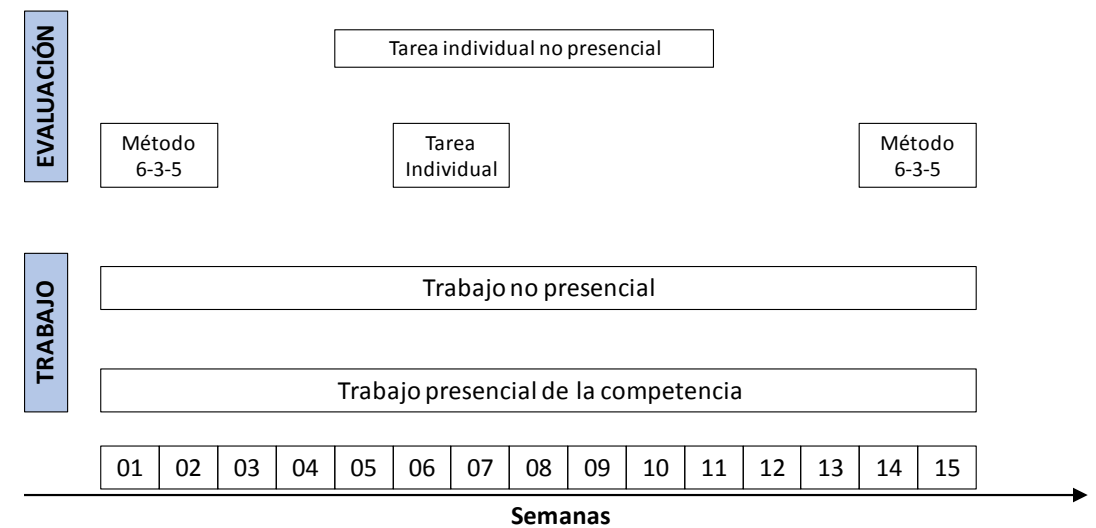

Fig. 3 Cronograma de la asignatura. Elaboración propia

La evaluación está centrada en cuatro actividades, de las cuales, tres son presenciales y una de ellas no presencial. Las actividades presenciales buscan que el alumno tenga que establecer la creación e innovación de ideas en un tiempo limitado (aproximadamente $1 \mathrm{~h}$ que dura la actividad). En cambio, la actividad de evaluación no presencial, dota al alumno de una mayor libertad de tiempo para desarrollar dicha actividad. Por tanto, dispone de un mayor tiempo para la generación y razonamiento de las ideas, las cuales pueden ser abordadas de una forma más pausada y ser consideradas en una mayor profundidad. Las tareas presenciales han sido el Método 6-3-5 (Cuenca et al., 2015), una al inicio de cuatrimestre y otra al final, así como una "Fast task", la cual es propuesta a mitad de cuatrimestre que coincide con la evaluación del primer parcial. En todos los casos, las tareas son adaptadas al curso de grado o máster, dependiendo donde se esté impartiendo. La tarea no presencial propuesta, contempla el mismo problema que debe ser abordado tanto por los alumnos de grado como de máster. El objetivo, es poder analizar las diferencias 
existentes entre las ideas aportadas por los diferentes alumnos en función del nivel de dominio donde se encuentren.

En cuanto al método 6-3-5, éste ha sido complementado de forma que el alumno además de trabajar la CT-04, permita trabajar y mejorar otras competencias transversales incluidas en el Plan Estrategico UPV2020 (Vidal-Carreras et al., 2016). La metodología desarrollada es descrita en la siguiente sección.

\subsection{Ejemplo de tarea desarrollada. Método 6-3-5 con decisión final}

Tal y como se ha descrito, dos de las actividades desarrolladas se corresponden con el Método 6-3-5. Este método es una técnica de creatividad derivada de la tormenta de ideas, en la cual, el alumno debe trabajar en grupo (Beitz et al., 1996). En esta actividad, los alumnos deben establecer grupos de 6 personas, los cuales deben escribir 3 ideas cada uno sobre una problemática planteada en un tiempo de $5 \mathrm{~min}$ cada uno. Esto permite establecer un total de 18 ideas en $30 \mathrm{~min}$.

Este metodo que presenta ventajas como la facilidad de desarrollo, no necesidad de un moderador cualificado y el incremento del aprendizaje activo por parte del estudiante. En cambio, tiene ciertas desventajas como la falta de creatividad por la limitación del tiempo o por falta de discusión y reflexión.

Cómo novedad en el método planteado, en la actividad además de establecer la metodología normal de desarrollo del método 6-3-5, fue introducida una actividad complementaria para trabajar tanto las competencias de comunicación efectiva como de trabajo en equipo y liderazgo.

De este modo, la metodología de trabajo durante el desarrollo de la clase fue:

1) Formación de grupos de trabajo de 6 personas (en el caso de máster de 4, porque el número era más reducido). En estos grupos de trabajo se evita que sean alumnos que habitualmente estudien o asistan juntos a clase en el mismo grupo. Con esta decisión, se fomenta el trabajo en equipo en ambientes de menor confianza.

2) Presentación del problema. El profesor a través de una imagen y/o pregunta, presenta el problema que en todos los casos (grado y máster) estaba relacionado con una unidad didáctica impartida en el curso. Por lo tanto, los alumnos pueden basarse en los resultados de aprendizaje de la asignatura así como otros alcanzados en otras asignaturas relacionadas o no con la Mecánica de los Fluidos.

3) Desarrollo del Método 6-3-5. Planteada la cuestión que engloba el problema, los alumnos forman los grupos de 6 personas, y tras seleccionar el turno de participación escribe cada uno de ellos sus tres ideas.

4) Fase de filtrado de ideas. Una vez el grupo ha desarrollado las 18 ideas, entre los seis integrantes deben de ponerse de acuerdo cual es la mejor idea, seleccionandola y argumentando su decisión en un máximo de 35 palabras, con ello se prentende que el alumno pueda sintetizar y plasmar las ideas, así como plantear de forma esquemática el plan acción en el caso de Nivel de Dominio III.

(cc) EY-NC-ND 2017, Universitat Politècnica de València 
5) Defensa de la idea seleccionada. Una vez el grupo ha decidido la idea, la ha justificado y ha nombrado un portavoz (todo ello en $5 \mathrm{~min}$ ), se genera un debate en clase, en el cual cada portavoz: enumera las 18 ideas, expone la idea seleccionada (durante un tiempo máximo de $3 \mathrm{~min}$, sin turno de réplica), argumentando porque la ha seleccionado frente a otras, así como plasmando las lineas de acción para solventar el problema (Nivel de Dominio III, únicamente). Finalmente, se realiza una votación a mano alzada en la cual se selecciona la mejor idea para solventar el problema.

\subsection{Evaluación de la competencia transversal}

La evaluación de la competencia transversal se ha planteado mediante el uso de rúbrica. La rúbrica utilizada ha sido adaptada por la propuesta desde el grupo de trabajo del Instituto de Ciencias de la Educación (Cuenca et al., 2015b) para ambos niveles. Está rúbrica ha sido adaptada atendiendo a las actividades desarrolladas en los cuatro indicadores tanto para el Nive I como Nivel III, tal y como se refleja en la Tabla 1 y Tabla 2 adjuntadas a continuación.

Tabla 1. Rúbrica de evaluación de las actividades para Nivel de dominio I.

\begin{tabular}{|c|c|c|c|c|}
\hline \multirow{2}{*}{ INDICADOR } & \multicolumn{4}{|c|}{ DESCRIPTOR } \\
\hline & D. No alcanzado & C. En desarrollo & B. Adecuado & A. Excelente \\
\hline $\begin{array}{l}\text { 1. Se cuestiona la } \\
\text { realidad }\end{array}$ & $\begin{array}{c}\text { No se cuestiona la } \\
\text { realidad }\end{array}$ & $\begin{array}{l}\text { Se plantea alguna } \\
\text { pregunta sobre la } \\
\text { realidad }\end{array}$ & $\begin{array}{c}\text { Aporta sus propias ideas } \\
\text { elaboradas con suficiente } \\
\text { detalle }\end{array}$ & $\begin{array}{l}\text { Aporta gran variedad de } \\
\text { ideas y planteamientos } \\
\text { novedosos, con fluidez } \\
\text { bien elaborados }\end{array}$ \\
\hline $\begin{array}{c}\text { Evaluación atendiendo a } \\
\text { la actividad }\end{array}$ & $\begin{array}{c}\text { El alumno no cuestiona } \\
\text { problema planteado en } \\
\text { la actividad }\end{array}$ & $\begin{array}{l}\text { El alumno cuestiona el } \\
\text { problema planteado en } \\
\text { la actividad, con } \\
\text { ejemplos planteados en } \\
\text { clase }\end{array}$ & $\begin{array}{c}\text { El alumno cuestiona el } \\
\text { problema planteado en la } \\
\text { actividad, detallando las } \\
\text { causas del mismo }\end{array}$ & $\begin{array}{l}\text { El alumno cuestiona el } \\
\text { problema planteado en la } \\
\text { actividad, detallando en } \\
\text { profundidad las causas } \\
\quad \text { del mismo }\end{array}$ \\
\hline 2. Aporta Ideas & $\begin{array}{c}\text { No aporta ideas y } \\
\text { muestra actitud pasiva } \\
\text { ante las tareas }\end{array}$ & $\begin{array}{l}\text { Plantea alguna idea ante } \\
\text { situaciones nuevas }\end{array}$ & $\begin{array}{c}\text { Aporta sus propias ideas, } \\
\text { elaborados con suficiente } \\
\text { detalle }\end{array}$ & $\begin{array}{c}\text { Aporta una gran variedad } \\
\text { de ideas y planteamientos } \\
\text { novedosos, con fluidez, } \\
\text { bien elaborados }\end{array}$ \\
\hline $\begin{array}{c}\text { Evaluación del } \\
\text { indicador atendiendo a } \\
\text { la actividad }\end{array}$ & $\begin{array}{l}\text { El alumno no aporta } \\
\text { ninguna idea, } \\
\text { determinando que el } \\
\text { problema no tiene } \\
\text { solución }\end{array}$ & $\begin{array}{c}\text { El alumno aporta pocas } \\
\text { ideas nuevas (1-2), } \\
\text { siendo algunas de } \\
\text { ejemplos dados en clase, } \\
\text { y en algunos casos que } \\
\text { no pueden solventar el } \\
\text { problema }\end{array}$ & $\begin{array}{l}\text { El alumno aporta ideas } \\
\text { (>3) relacionadas con la } \\
\text { mecánica de los fluidos. } \\
\text { En este caso, la mayoria } \\
\text { de las ideas resuelven la } \\
\text { problemática planteada. }\end{array}$ & $\begin{array}{l}\text { El alumno aporta ideas } \\
\text { relacionadas con la } \\
\text { mecánica de los fluidos y } \\
\text { las relaciona con } \\
\text { conceptos desarrollados } \\
\text { en otras aplicaciones } \\
\text { (>3). En este caso todas } \\
\text { las ideas resuelven la } \\
\text { problemática planteada }\end{array}$ \\
\hline $\begin{array}{l}\text { 3. Plasma de manera } \\
\text { formal las ideas }\end{array}$ & $\begin{array}{l}\text { No plasma de manera } \\
\text { formal las ideas }\end{array}$ & $\begin{array}{c}\text { Plasma de manera } \\
\text { limitada las ideas que se } \\
\text { han generado }\end{array}$ & $\begin{array}{c}\text { Plasma de manera } \\
\text { correcta las ideas que se } \\
\text { han generado }\end{array}$ & $\begin{array}{c}\text { Plasma de manera } \\
\text { enriquecida las ideas que } \\
\text { se han generado }\end{array}$ \\
\hline $\begin{array}{c}\text { Evaluación del } \\
\text { indicador atendiendo a } \\
\text { la actividad }\end{array}$ & $\begin{array}{c}\text { El alumno no aporta ni } \\
\text { plasma ninguna idea } \\
\text { para solucionar el } \\
\text { problema }\end{array}$ & $\begin{array}{l}\text { El alumno aporta ideas, } \\
\text { pero las plasma de una } \\
\text { manera confusa, como } \\
\text { consecuencia que no } \\
\text { resuelven el problema }\end{array}$ & $\begin{array}{c}\text { El alumno plasma las } \\
\text { ideas, fundamentándose } \\
\text { en los conceptos } \\
\text { desarrollados en la } \\
\text { asignatura }\end{array}$ & $\begin{array}{l}\text { El alumno plasma las } \\
\text { ideas, fundamentándose } \\
\text { en los conceptos } \\
\text { desarrollados en la } \\
\text { asignatura, así como en } \\
\text { otros conceptos } \\
\text { desarrollados en otras }\end{array}$ \\
\hline $\begin{array}{l}\text { 4. Identifica los } \\
\text { resultados }\end{array}$ & No identifica resultados & $\begin{array}{c}\text { Identifica de manera } \\
\text { limitada resultados }\end{array}$ & $\begin{array}{c}\text { Identifica adecuadamente } \\
\text { resultados }\end{array}$ & $\begin{array}{c}\text { Define parámetros } \\
\text { asociados a los resultados }\end{array}$ \\
\hline
\end{tabular}


Evaluar la competencia "Innovación, Creatividad y Emprendimiento" en asignaturas del área de la Mecánica de los Fluidos. Caso de estudio en Grado y Máster

\begin{tabular}{|c|c|c|c|c|}
$\begin{array}{c}\text { Evaluación del } \\
\text { indicador atendiendo a }\end{array}$ & $\begin{array}{c}\text { El alumno no puede dar } \\
\text { una solución a la } \\
\text { problemática planteada }\end{array}$ & $\begin{array}{c}\text { El alumno no tiene gran } \\
\text { variedad de resultados } \\
\text { para poder solventar la } \\
\text { problemática, como } \\
\text { consecuencia del número } \\
\text { reducido de ideas }\end{array}$ & $\begin{array}{c}\text { El alumno tiene posibles } \\
\text { resultados que ofrecer, } \\
\text { relacionados únicamente } \\
\text { con la asignatura }\end{array}$ & $\begin{array}{c}\text { El alumno tiene diferentes } \\
\text { resultados válidos que } \\
\text { todos ellos podrían dar } \\
\text { solución al problema, } \\
\text { relacionando no solo con } \\
\text { la asignatura sino con } \\
\text { otras asignaturas ya } \\
\text { cursadas }\end{array}$ \\
\end{tabular}

Tabla 2. Rúbrica de evaluación de las actividades para Nivel de dominio III.

\begin{tabular}{|c|c|c|c|c|}
\hline \multirow{2}{*}{ INDICADOR } & \multicolumn{4}{|c|}{ DESCRIPTOR } \\
\hline & D. No alcanzado & C. En desarrollo & B. Adecuado & A. Excelente \\
\hline $\begin{array}{c}\text { 1. Integra } \\
\text { conocimientos de otras } \\
\text { disciplinas }\end{array}$ & $\begin{array}{l}\text { No es capaz de integrar } \\
\text { sus conocimientos } \\
\text { previos para generar } \\
\text { nuevos conocimientos o } \\
\text { ideas }\end{array}$ & $\begin{array}{l}\text { Integra conocimientos de } \\
\text { diversos campos pero sin } \\
\text { que ello sirva para } \\
\text { generar ideas novedosas }\end{array}$ & $\begin{array}{l}\text { Genera nuevas ideas } \\
\text { integrando conocimientos } \\
\text { de varias disciplinas, } \\
\text { fuentes o ambitos }\end{array}$ & $\begin{array}{c}\text { La integración que realiza } \\
\text { de conocimientos genera } \\
\text { ideas que mejoran las } \\
\text { soluciones anteriores a un } \\
\text { problema o situación } \\
\text { determinada }\end{array}$ \\
\hline $\begin{array}{c}\text { Evaluación atendiendo a } \\
\text { la actividad }\end{array}$ & $\begin{array}{c}\text { El alumno no cuestiona } \\
\text { el problema planteado en } \\
\text { la actividad ni aporta } \\
\text { ideas }\end{array}$ & $\begin{array}{c}\text { El alumno cuestiona el } \\
\text { problema planteado en } \\
\text { la actividad, aportando } \\
\text { alguna idea desarrollada } \\
\text { en clase }\end{array}$ & $\begin{array}{l}\text { El alumno cuestiona el } \\
\text { problema planteado en la } \\
\text { actividad, detallando las } \\
\text { causas del mismo y } \\
\text { aportando ideas } \\
\text { relacionadas con la } \\
\text { asignatura }\end{array}$ & $\begin{array}{l}\text { El alumno cuestiona el } \\
\text { problema planteado en la } \\
\text { actividad, detallando en } \\
\text { profundidad las causas } \\
\text { del mismo y aportando } \\
\text { ideas relacionada con la } \\
\text { asignatura y otras } \\
\text { diferentes ya cursadas }\end{array}$ \\
\hline $\begin{array}{l}\text { 2. Adopta enfoques } \\
\text { creativos en el } \\
\text { contenido y modo de } \\
\text { realización }\end{array}$ & $\begin{array}{c}\text { No propone ideas } \\
\text { originales a la situación } \\
\text { planteada }\end{array}$ & $\begin{array}{l}\text { Propone ideas y enfoques } \\
\text { que no se adaptan a la } \\
\text { situación y/o no } \\
\text { especifica correctamente } \\
\text { el modo de ejecución }\end{array}$ & $\begin{array}{c}\text { Adopta enfoques } \\
\text { adecuados de la situación } \\
\text { y un correcto } \\
\text { planteamiento del modo } \\
\text { de realización }\end{array}$ & $\begin{array}{c}\text { Adopta enfoques } \\
\text { originales, genera nuevas } \\
\text { ideas divergentes a partir } \\
\text { de perspectivas diferentes } \\
\text { y aporta creatividad en lo } \\
\text { que hace, mejorando } \\
\text { sistemas, procedimientos } \\
\text { y procesos }\end{array}$ \\
\hline $\begin{array}{c}\text { Evaluación del } \\
\text { indicador atendiendo a } \\
\text { la actividad }\end{array}$ & $\begin{array}{l}\text { El alumno no aporta } \\
\text { ninguna idea, } \\
\text { determinando que el } \\
\text { problema no tiene } \\
\text { solución }\end{array}$ & $\begin{array}{c}\text { El alumno aporta pocas } \\
\text { ideas nuevas (l), siendo } \\
\text { algunas de ejemplos } \\
\text { dados en clase, y en } \\
\text { algunos casos que no } \\
\text { pueden solventar el } \\
\text { problema }\end{array}$ & $\begin{array}{l}\text { El alumno aporta ideas } \\
\text { relacionadas con la } \\
\text { mecánica de los fluidos } \\
\text { (2-3). En este caso, la } \\
\text { mayoría de las ideas } \\
\text { resuelven la problemática } \\
\text { planteada. }\end{array}$ & $\begin{array}{l}\text { El alumno aporta ideas } \\
\text { relacionadas con la } \\
\text { mecánica de los fluidos y } \\
\text { las relaciona con } \\
\text { conceptos desarrollados } \\
\text { en otras aplicaciones } \\
\text { (>3). En este caso todas } \\
\text { las ideas resuelven la } \\
\text { problemática planteada }\end{array}$ \\
\hline $\begin{array}{l}\text { 3. Propone un plan de } \\
\text { acción }\end{array}$ & $\begin{array}{l}\text { No genera una lista } \\
\text { completa de tareas }\end{array}$ & $\begin{array}{c}\text { Genera una lista de } \\
\text { tareas que establezca el } \\
\text { plan de acción pero falta } \\
\text { información relevante }\end{array}$ & $\begin{array}{l}\text { Plantea de forma completa } \\
\text { un plan de acción }\end{array}$ & $\begin{array}{c}\text { Plantea de manera formal } \\
\text { y detallada un plan de } \\
\text { acción, ejecutando alguna } \\
\text { de sus fases }\end{array}$ \\
\hline $\begin{array}{c}\text { Evaluación del } \\
\text { indicador atendiendo a } \\
\text { la actividad }\end{array}$ & $\begin{array}{c}\text { El alumno no es capaz de } \\
\text { generar una lista de } \\
\text { tareas para desarrollar } \\
\text { su plan }\end{array}$ & $\begin{array}{l}\text { El alumno es capaz de } \\
\text { generar una lista de } \\
\text { tareas, al menos sus } \\
\text { puntos fundamentales, } \\
\text { pero no es capaz de } \\
\text { completar las variables } \\
\text { directos e indirectos que } \\
\text { afectan al problema }\end{array}$ & $\begin{array}{c}\text { El alumno completa el } \\
\text { plan de acción con la } \\
\text { información necesaria } \\
\text { para desarrollarlo, } \\
\text { describiendo la totalidad } \\
\text { de variables directas e } \\
\text { indirectas que afectan a la } \\
\text { solución de la } \\
\text { problemática }\end{array}$ & $\begin{array}{c}\text { El alumno completa el } \\
\text { plan de acción, define la } \\
\text { totalidad de las variables } \\
\text { y es capaz de llevarlo en } \\
\text { parte o en su totalidad, } \\
\text { sino de forma práctica a } \\
\text { través de una descripción } \\
\text { teórica. }\end{array}$ \\
\hline $\begin{array}{l}\text { 4. Analiza el valor de la } \\
\text { innovación }\end{array}$ & $\begin{array}{c}\text { No lleva a cabo ningún } \\
\text { análisis }\end{array}$ & $\begin{array}{l}\text { Identifica algunos } \\
\text { riesgos de la innovación } \\
\text { de forma limitada }\end{array}$ & $\begin{array}{l}\text { Realiza algun análisis del } \\
\text { valor de la innovación }\end{array}$ & $\begin{array}{c}\text { Realiza un análisis global } \\
\text { del valor de la innovación, } \\
\text { utilizando herramientas } \\
\text { adecuadas }\end{array}$ \\
\hline $\begin{array}{c}\text { Evaluación del } \\
\text { indicador atendiendo a }\end{array}$ & $\begin{array}{l}\text { El alumno no aporta } \\
\text { idea o si la aporta no }\end{array}$ & $\begin{array}{l}\text { El alumno identifica al } \\
\text { menos un punto débil de }\end{array}$ & $\begin{array}{l}\text { El alumno identifica los } \\
\text { puntos fuertes y débiles de }\end{array}$ & $\begin{array}{l}\text { El alumno identifica los } \\
\text { puntos fuertes y débiles de }\end{array}$ \\
\hline
\end{tabular}

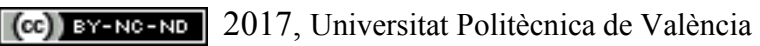

Congreso IN-RED (2017) 


\begin{tabular}{|c|c|c|c|c|}
\hline la actividad & $\begin{array}{l}\text { puede desarrollar un } \\
\text { análisis de la solución a } \\
\text { problemática planteada }\end{array}$ & $\begin{array}{l}\text { su innovación o idea, no } \\
\text { siendo capaz de } \\
\text { identificar los riesgos } \\
\text { que conlleva }\end{array}$ & $\begin{array}{l}\text { la innovación (>3), siendo } \\
\text { críco con aspectos } \\
\text { relacionados con la } \\
\text { mecánica de los fluidos }\end{array}$ & $\begin{array}{l}\text { la innovación (>5), siendo } \\
\text { crítico con aspectos } \\
\text { relacionados con la } \\
\text { mecánica de los fluidos } \\
\text { así como con aspectos de } \\
\text { su entorno de los cuales } \\
\text { no depende la mecánica } \\
\text { de fluidos }\end{array}$ \\
\hline
\end{tabular}

Definidas las rúbricas, adaptando el indicador a la evaluación de la actividad realizada, y teniendo en cuenta que es el primer año que se desarrollaba la actividad y se evaluaba la competencia, los autores decidieron otorgar un mayor peso escecifico al indicador 1 y 2 de cada uno de las rúbricas. Ambos indicadores compuntan un $60 \%$ (30\% cada uno) de la evaluación de la competencia y los indicadores 3 y 4 de cada rúbrica un $20 \%$ cada uno.

Cómo existian 4 actividades diferentes, se desarrolló una equiparación númerica a cada una de los grados alcanzados en cada uno de los indicadores y actividades, para posteriormente, poder establecer el grado final alcanzado por el alumno. La equivalencia númerica fues establecida de acuerdo a la Tabla 3.

Tabla 3. Evaluación de competencia transversal.

\begin{tabular}{|c|c|}
\hline Grado Alcanzado en el Indicador & Equivalencia Númerica \\
\hline Excelente (A) & 5 \\
\hline Bien (B) & 2 \\
\hline En desarrollo (C) & 1 \\
\hline No alcanzado (D) & 3 \\
\hline
\end{tabular}

Definida cada actividad como $\left(\mathrm{A}_{\mathrm{i}}\right)$, la nota obtenida en la competencia transversal viene definida por la expresión:

$$
N C=\frac{\sum_{i=1}^{n}\left(0.3 * I_{1}+0.3 * I_{2}+0.2 * I_{3}++0.2 * I_{4}\right)}{n}
$$

Donde NC es la nota numérica obtenida en la evaluación de la competencia transversal; $I_{1}$ es el nivel alcanzado en el indicador $1 ; I_{2}$ es el nivel alcanzado en el indicador $2 ; \mathrm{I}_{3}$ es el nivel alcanzado en el indicador $3 ; \mathrm{I}_{4}$ es el nivel alcanzado en el indicador 4; y n es el número de actividades desarrolladas.

La evaluación de la competencia viene establecida según la Tabla 4:

Tabla 4. Evaluación de competencia transversal.

\begin{tabular}{|c|c|}
\hline Evaluación Competencia & Equivalencia Númerica \\
\hline Excelente (A) & $3.5 \leq \mathrm{NC} \leq 5$ \\
\hline Bien (B) & $2 \leq \mathrm{NC}<3.5$ \\
\hline
\end{tabular}

(cc)) BY-NC-ND 2017, Universitat Politècnica de València 


\begin{tabular}{|c|c|}
\hline En desarrollo (C) & $1<N C<2$ \\
\hline No alcanzado (D) & 1 \\
\hline
\end{tabular}

\section{Resultados}

La metodología descrita se ha puesto en marcha en el curso 2016/17, y ambas asignaturas se imparten en el cuatrimestre B. Por lo tanto, no se pueden mostrar los resultados finales de la evaluación final de los alumnos, pero sí que se pueden mostrar resultados parciales de evaluación, así como los resultados obtenidos en el Método 6-3-5. Aunque son resultados parciales, muestran un buen grado de satisfación tanto en los resultados académicos como en la opinión personal de los alumnos que han desarrollado las actividades.

\subsection{Resultados Método 6-3-5}

Como ejemplo, se adjunta la pregunta (Figura 4) que fue planteada en la primera actividad del Método 6-3-5 a los alumnos de Grado. La asignatura se denomina Mecánica de Fluidos es troncal de segundo curso de Grado de Ingeniería Química. En ella el profesor mostraba una imagen de un jugador de baloncesto que se disponía a saltar sobre una piscina llena de fluido. El objetivo del jugador era realizar un "mate" en una canasta cuya proyección se encontraba dentro de la piscina. Para alcanzar el objetivo, el jugador debía saltar sobre el fluido para poder alcanzar la canasta.

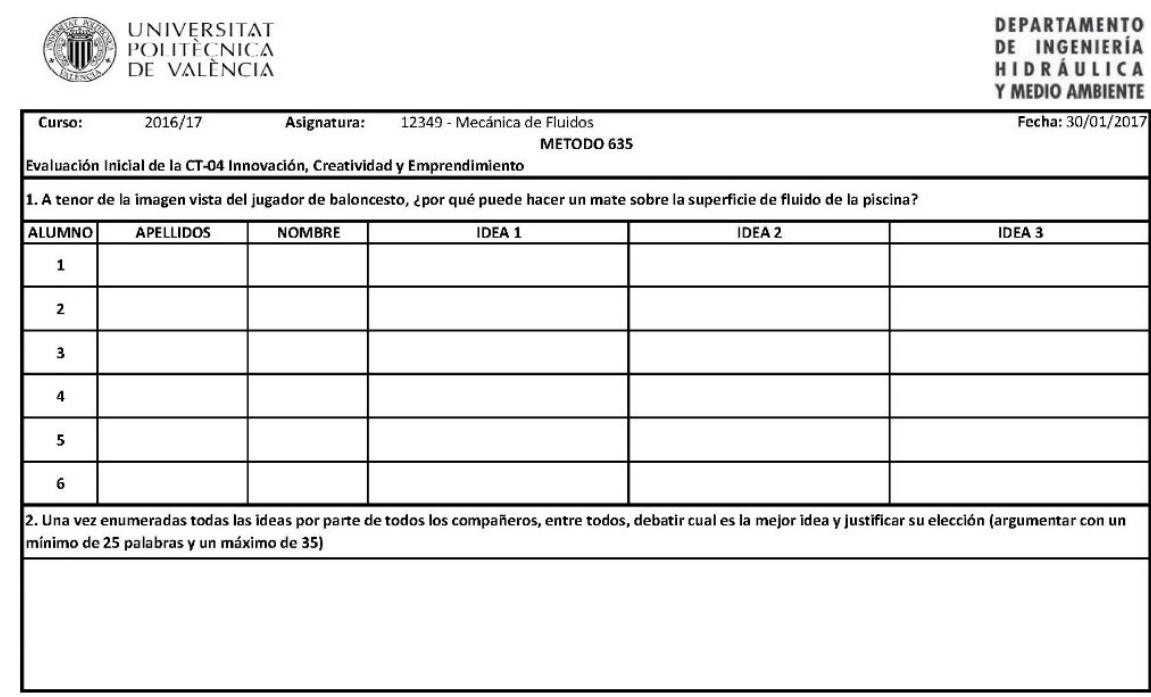

Fig. 4 Plantilla aportada a cada grupo

En la experiencia participaron 30 alumnos formandose 5 grupos diferentes. El número total de ideas desarrolladas fueron 90 en un tiempo total de $30 \mathrm{~min}$. De ese número de ideas únicamente el 13.33\% (12 de 90 fueron repetidas por el conjunto de alumnos). Una vez propuestas las ideas, se debatieron en grupo y seleccionaron una idea que posteriormente 
expusieron. De las 5 ideas seleccionadas, 3 de ellas dieron con la solución correcta del problema. Evaluando posteriormente las ideas aportadas por cada alumno y grupo, mediante la rubrica adjuntada en la Tabla 1, se obtuvieron los siguientes resultados en los diferentes indicadores (adjuntados en la Tabla 5). Los resultados obtenidos de los indicadores 3 y 4 son resultados que todos los miembros del grupo tenían la misma nota, como consecuencia de que fue analizado y debatido en grupo.

Tabla 5. Resultados de la evaluación del Método 6-3-5 aplicado a la asignatura de Grado.

\begin{tabular}{|c|c|c|c|c|}
\hline \multirow{2}{*}{ GRADO ALCANZADO } & \multicolumn{4}{|c|}{ INDICADOR } \\
\cline { 2 - 5 } & $\mathbf{I 1}$ & $\mathbf{I 2}$ & $\mathbf{I 3}$ & $\mathbf{I 4}$ \\
\hline $\mathbf{A}$ & $66.67 \%$ & $63.33 \%$ & $80.00 \%$ & $40.00 \%$ \\
\hline $\mathbf{B}$ & $33.33 \%$ & $30.00 \%$ & $20.00 \%$ & $40.00 \%$ \\
\hline $\mathbf{C}$ & $0.00 \%$ & $6.67 \%$ & $0.00 \%$ & $20.00 \%$ \\
\hline $\mathbf{D}$ & $0.00 \%$ & $0.00 \%$ & $0.00 \%$ & $0.00 \%$ \\
\hline
\end{tabular}

En cuanto a la experiencia desarrollada en los alumnos de Master, las características de la asignatura, con solamente 8 participantes, hacen que la participación sea completa y con una especificidad mucho mayor.

La asignatura se denomina Dispersión de Contaminantes en Aire y Atmósfera y es troncal de intensificación de segundo curso de Master en Ingeniería Industrial en la especialidad de Medio Ambiente. En este caso, el método en lugar de realizarse en grupos de 6 alumnos se realizó unicamente con dos grupos de 4 alumnos.

La ficha entregada a los mismos fue idéntica a la presentada en la Figura 1, con la siguiente pregunta: ¿Por qué la alcaldesa de Madrid no permite la entrada al tráfico de los coches durante ciertos días al año?. La participación fue completa y se generaron 24 ideas, algunas de ellas con verdadera innovación sobre la respuesta. La pregunta se ha realizado en el primer contacto con los alumnos, cuando aún no tienen los conocimientos adquiridos, y la experiencia además es muy significativa del grado de conocimientos previos adquiridos.

En este caso particular, la verdadera solución con una explicación científica profunda requería de un conocimiento muy específico de la materia, que ninguno de los alumnos había adquirido previamente. Sin embargo, en la votación, todos coincidieron en la misma idea como la más verosimil y en verdad, la más cercana a la solución final.

Cabe decir que a lo largo de la asignatura se introducen los conceptos necesarios para la correcta respuesta de la misma; y realizada la misma pregunta en semanas posteriores, la respuesta sí es la correcta en todos los casos. El aprendizaje en este caso ha sido completo.

\section{Conclusiones}

En cuanto a las conclusiones obtenidas en la experiencia desarrollada, se pueden enumerar las siguientes conclusiones: 
1) Se ha conseguido despertar el interes de los alumnos (tanto de Grado como de Máster) en temas poco seductores para su estudio, como puede ser el análisis de la propiedades de fluidos no newtonianos, que en otros tipos de aprendizaje más deductivo no se muestran tan paticipativos.

2) La limitación del tiempo en la creacción de ideas muestra que no es un factor limitante en el desarrollo del aprendizaje del alumno. Según las experiencias desarrolladas, los alumnos son capaces de generar un gran número de ideas con un bajo índice de repeticiones entre todos los alumnos en un breve periodo de tiempo.

3) Se ha desarrollado una rubrica de evaluación con base a los indicadores y descriptores propuestos desde el Instituto de Ciencias y de la Educación, la cual ha sido adaptada a ambas asignaturas, impartidas tanto a nivel I como nivel III, en función de las actividades propuestas.

4) Para permitir trabajar al alumno en diferentes ambientes, se han propuesto: (i) dos tareas presenciales grupales del Método 6-3-5, incorporando la discusión y comunicación en la actividad; (ii) una tarea presencial que el alumno de forma individual en un tiempo corto $(10 \mathrm{~min})$ debe proponer ideas y realizar un plan de acción a una problemática planteada; y (iii) una tarea no presencial en la cual el alumno, cuenta con más tiempo (1 mes) para desarrollarla. En esta tarea, el alumno debe: identificar el problema, aportar ideas, identificar ventajas e inconvenientes, relacionandolo con otros posibles afecciones colaterales y seleccionando la mejor idea que considera.

5) Dos rúbricas han sido propuestas para la evaluación de actividades que esten incluidas dentro de la evaluación. Del mismo modo, se ha propuesto una expresión matemática que permita combinar las evaluaciones de los indicadores en las distintas actividades. El establecimiento de la nota final de la competencia (grado alcanzado) es desarrollado considerando un peso ponderado de cada uno de los indicadores.

6) La rúbrica planteada y método de evaluación se puede adaptar a otras asignaturas $\mathrm{u}$ otras actividades en próximos cursos, en los cuales se trabajen los indicadores de forma diferente.

7) Se han mostrado resultados parciales de dicha evaluación, teniendo en cuenta las actividades de generación de ideas que sí se han realizado.

Finalmente, la técnica se ha mostrado muy motivadora para los alumnos tanto de Grado como de Máster. Los autores de la presente investigación perciben una buena acogida de la actividad, así como unos resultados satisfactorios en cuanto a la fijación de los conceptos finales en los alumnos. Esto convierte a la metodología desarrolada en una estrategia motivadora para el alumnado, en su proceso de aprendizaje de conceptos complejos.

\section{Referencias}

Beitz, G. P. W., Wallace, K., Blessing, L., Bauert, F. (1996). Engineering Design: A Systeatic Approach.MRS BULLETIN, 71.

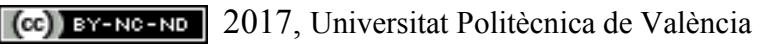


Cuenca, L., Alarcon, F., Boza, A., Fernández-Diegno, M., Ruíz, L., Gordo, ML., Poler, R., Alemany, M.M.E. (2015). "Rúbrica para la Evaluación de la Competencia Innovación, Creatividad y Emprendimiento en máster”. Congreso In-Red 2015-Universitat Politècnica de València. ISBN: 978-84-9048-396-1. Disponible en < http://ocs.editorial.upv.es/index.php/INRED/INRED2015>

Cuenca, L., Bonet Espinosa, M.P., Boza Garcia, A., Fuentes-Durá, P., LajaraCAMILleri, N., MARÍN GARcÍA, JA., Peris-OrTIZ, M. (2015). Innovation, Creativity and Entreprenaeurship Learning Outcomes in Higher Education. IATED, International Association of Technology, Education and Development. http://hdl.handle.net/10251/72847.

Fernandes, J.M., Afonso, P., Fonte, V., Alves, V., Ribeiro, A.N. (2017) Promoting entrepreneurship among informatics engineering students:insights from a case study. European Journal of Engineering Education, 42:1, 91-108, DOI: 10.1080/03043797.2016.1197891

LÓPEZ-JIMÉNEZ, P.A., PÉREZ-SÁNCHEZ, M. "Los objetos de aprendizaje como conductores para favorecer el aprendizaje autónomo en el campo de la mecánica de fluidos”. II Congreso Nacional de Innovación Educativa y de Docencia en Red (Valencia, 7 y 8 de julio, 2016). Editorial Universitat Politècnica de València, 2016. Doi:http://dx.doi.org/10.4995/INRED2016.2016.4347

PÉREZ-SÁnCHEZ, M., LÓPEZ-JiMÉnEZ, P.A. (2017) “The Use of Learning Objects in Environmental Education: The Experience of Distance Asynchronous Teaching in Pollutant Dispersion Topics". Environmental Education: Perspectives, Challenges and Opportunities

RAE, Real Academia Española de la Lengua http://www.rae.es/ [Consulta 14 de marzo de 2017]

VidAl-CARreras, P.I., CANOS-DAROS, L., GARCÍA-SABATER, J.P. "Marcadores para una herramienta de evaluación de Wikis". II Congreso Nacional de Innovación Educativa y de Docencia en Red (Valencia, 7 y 8 de julio, 2016). Editorial Universitat Politècnica de València, 2016. Doi:http://dx.doi.org/10.4995/INRED2016.2016.4347 